Communications in Nonlin. Sci. and Numer. Simulation, (2006) doi:101016/j.cnsns.2006.06.015 


\title{
An inverse problem with data on the part of the boundary
}

\author{
A.G. Ramm \\ Mathematics Department, Kansas State University, \\ Manhattan, KS 66506-2602, USA \\ ramm@math.ksu.edu
}

\begin{abstract}
Let $u_{t}=\nabla^{2} u-q(x) u:=L u$ in $D \times[0, \infty)$, where $D \subset R^{3}$ is a bounded domain with a smooth connected boundary $S$, and $q(x) \in L^{2}(S)$ is a real-valued function with compact support in $D$. Assume that $u(x, 0)=0, u=0$ on $S_{1} \subset S, u=a(s, t)$ on $S_{2}=S \backslash S_{1}$, where $a(s, t)=0$ for $t>T, a(s, t) \not \equiv 0, a \in C^{1}\left([0, T] ; H^{3 / 2}\left(S_{2}\right)\right)$ is arbitrary.

Given the extra data $\left.u_{N}\right|_{S_{2}}=b(s, t)$, for each $a \in C^{1}\left([0, T] ; H^{3 / 2}\left(S_{2}\right)\right)$, where $N$ is the outer normal to $S$, one can find $q(x)$ uniquely. A similar result is obtained for the heat equation $u_{t}=\mathcal{L} u:=\nabla \cdot(a \nabla u)$.

These results are based on new versions of Property $\mathrm{C}$.
\end{abstract}

\section{Introduction}

Let $D \subset \mathbb{R}^{3}$ be a bounded domain with a smooth connected boundary $S, S=S_{1} \cup S_{2}$, $S_{1}$ is an open subset in $S$, and $S_{2}$ is the complement of $S_{1}$ in $S$. Consider the problem

$$
\begin{aligned}
& u_{t}=\nabla^{2} u-q(x) u, \quad(x, t) \in D \times[0, \infty), \\
& u(x, 0)=0,\left.\quad u\right|_{S_{1}}=0,\left.\quad u\right|_{S_{2}}=a(s, t),
\end{aligned}
$$

where $q(x) \in L^{2}(D)$ is a real-valued function, $a(s, t) \in C^{1}\left([0, \infty), H^{3 / 2}\left(S_{2}\right)\right)$, the function $a$ is an arbitrary real-valued function in the above set such that $a \not \equiv 0, a=0$ for $t>T$, $H^{\ell}$ is the Sobolev space.

For each $a$ in the above set let the extra data be given:

$$
\left.u_{N}\right|_{S_{2}}=b(s, t),
$$

MSC: 35K20, 35R30; PACS 02.30.Jr

Key words: Property C, parabolic equations, inverse problems 
where $N$ is the outer normal to the boundary $S$.

Do the data $\{a(s, t), b(s, t)\} \forall a(s, t) \in C^{1}\left([0, \infty), H^{3 / 2}\left(S_{2}\right)\right), a \not \equiv 0, a=0$ for $t>T$, determine $q(x)$ uniquely?

Our main result is a positive answer to this question.

Theorem 1. The data $\{a(s, t), b(s, t)\}_{\forall t \geq 0, \forall s \in S_{2}}$, given for all a with the above properties, determine a compactly supported in D real-valued function $q(x) \in L^{2}(D)$ uniquely.

Actually we prove a slightly stronger result: the data for $0 \leq t \leq T+\varepsilon$ determine $q$ uniquely, where $\varepsilon>0$ is an arbitrary small number.

Note that the set $S_{2}$ can be arbitrary small.

Theorem 1 is a multidimensional generalization of the author's result from [1]. Let

$$
L_{j} v-\lambda v:=\nabla^{2} v-q_{j}(x) v-\lambda v, \quad j=1,2
$$

and

$$
N_{j}=\left\{v: L_{j} v-\lambda v=0 \text { in } D,\left.v\right|_{S_{1}}=0\right\} .
$$

The main tool in the proof of Theorem 1 is a new version of property C. Originally this property was introduced by the author in 1986 for the products of solutions to homogeneous linear partial differential equations in the case when these solutions did not satisfy any boundary conditions (see [3]).

Theorem 2. The set $\left\{v_{1}, v_{2}\right\}$ for all $v_{1} \in N_{1}$ and all $v_{2} \in N_{2}$ is complete (total) in $L^{2}\left(D_{1}\right)$, where $D_{1} \subset D$ is a strictly inner subdomain of $D$, i.e., if $p \in L^{2}\left(D_{1}\right), p=0$ in $D \backslash D_{1}$, and $\int_{D_{1}} p v_{1} v_{2} d x=0 \quad \forall v_{1} \in N_{1}, \forall v_{2} \in N_{2}$, then $p=0$.

In Section 2 Theorems 1 and 2 are proved. In Section 3 the results are generalized to the boundary-value problems for the equation $u_{t}=\nabla \cdot(a(x) \nabla u)$.

\section{Proofs}

Proof of Theorem 1. Let

$$
v=\int_{0}^{\infty} e^{-\lambda t} u d t \quad \text { and } \quad A(s, \lambda)=\int_{0}^{\infty} e^{-\lambda t} a(s, t) d t
$$

Taking the Laplace transform of the relations (1)-(2), we obtain:

$$
L v-\lambda v=0, \quad L v=\nabla^{2} v-q(x) v,\left.\quad v\right|_{S_{1}}=0,\left.\quad v\right|_{S_{2}}=A(s, \lambda),
$$

and

$$
v_{N}=B(s, \lambda)=\int_{0}^{\infty} e^{-\lambda t} b(s, t) d t .
$$


Assume that there are $q_{1}$ and $q_{2}$, compactly supported in $D$, which generate the same data. Let

$$
L_{j} v_{j}=\nabla^{2} v_{j}-q_{j} v_{j}, \quad j=1,2,
$$

and

$$
w=v_{1}-v_{2} .
$$

Then

$$
L_{1} w-\lambda w=p v_{2}, \quad p=q_{1}-q_{2},
$$

and for any

$$
\psi \in N\left(L_{1}-\lambda\right):=\left\{\psi:\left(L_{1}-\lambda\right) \psi=0\right\},\left.\quad \psi\right|_{S_{1}}=0,
$$

one gets

$$
\int_{D} p v_{2} \psi d x=\int_{S_{2}}\left(w_{N} \psi-\psi_{N} w\right) d s=0
$$

because

$$
w=\psi=0 \quad \text { on } \quad S_{1}
$$

and

$$
w=w_{N}=0 \quad \text { on } \quad S_{2}
$$

by our assumptions. By Theorem 2, relation (6) implies $p=0$.

Theorem 1 is proved.

Proof of Theorem 2. It is proved in [2] (see also [4]) that the set $\left\{v_{1} v_{2}\right\}$ for all

$$
v_{j} \in M_{j}:=\left\{v_{j}:\left(L_{j}-\lambda\right) v_{j}=0 \text { in } D\right\}
$$

is total in $L^{2}(D)$. Therefore it is sufficient to prove that $N_{j}$ is dense in $M_{j}$ in $L^{2}\left(D_{1}\right)$-norm, where $D_{1}$ is a strictly inner subdomain of $D$ out of which both $q_{1}$ and $q_{2}$ vanish.

Let us take $j=1$. The proof for $j=2$ is the same. Assume the contrary. Then there is a $\psi \in M_{1}$ such that

$$
0=\int_{D_{1}} \psi v d x \quad \forall v \in N_{1} .
$$

Let $G(x, y)$ solve the problem

$$
\left(L_{1}-\lambda\right) G=-\delta(x-y) \text { in } \mathbb{R}^{3}, \quad \lim _{|x-y| \rightarrow \infty} G(x, y)=0,\left.\quad G\right|_{S_{1}}=0 .
$$

Since $G(x, y) \in N_{1} \forall y \in D_{1}^{\prime}=\mathbb{R}^{3} \backslash D_{1}$, equation (7) implies

$$
0=\int_{D_{1}} \psi(x) G(x, y) d x:=h(y), \quad \forall y \in D_{1}^{\prime} .
$$

We have $h(y) \in H_{\text {loc }}^{2}\left(\mathbb{R}^{3} \backslash S_{1}\right)$, and $h$ solves the elliptic equation

$$
\left(\nabla^{2}-\lambda\right) h=0 \quad \text { in } \quad D_{1}^{\prime},
$$


because $q_{1}=q_{2}=0$ in $D_{1}^{\prime}$. Therefore, one gets from (9) the following relations:

$$
h=h_{N}=0 \text { on } \partial D_{1},
$$

and, because of (8), one gets

$$
\left(L_{1}-\lambda\right) h=-\psi \text { in } D_{1} .
$$

Multiply (11) by $\bar{\psi}$, integrate over $D_{1}$, use (10), and get

$$
-\int_{D_{1}}|\psi|^{2} d x=\int_{\partial D_{1}}\left(h_{N} \bar{\psi}-h \overline{\psi_{N}}\right) d s=0 .
$$

Thus $\psi=0$ in $D_{1}$ and, therefore, $\psi=0$ in $D$, because $\psi$ solves a homogeneous linear elliptic equation for which the uniqueness of the solution to the Cauchy problem holds. Theorem 2 is proved.

\section{Generalizations}

Consider now the problem

$$
\begin{gathered}
u_{t}=\nabla \cdot(a(x) \nabla u):=\mathcal{L} u, \quad(x, t) \in D \times[0, \infty), \\
u(x, 0)=0,\left.\quad u\right|_{S_{1}}=0,\left.\quad u\right|_{S_{2}}=h(s, t),
\end{gathered}
$$

and the extra data are

$$
\left.a u_{N}\right|_{S_{2}}=z(s, t) \quad \forall s \in S_{2}, \forall t>0 .
$$

We assume that

$$
0<a_{0} \leq a(x) \leq a_{1},
$$

where $a_{0}$ and $a_{1}$ are constants, $a \in H^{3}(D), h \in C^{1}\left([0, \infty), H^{3 / 2}\left(S_{2}\right)\right)$ is arbitrary, and prove the following theorem.

Theorem 3. Under the above assumptions, the data $\{h(s, t), z(s, t)\}_{\forall s \in S_{2}, \forall t>0, \forall h}$ determine a $(x)$ uniquely.

Proof. Taking the Laplace transform of relations (13)-(15), we reduce the problem to

$$
(\mathcal{L}-\lambda) v=0 \text { in } D,\left.\quad v\right|_{S_{1}}=0,\left.v\right|_{S_{2}}=H(s, \lambda),\left.\quad a v_{N}\right|_{S_{2}}=Z(s, \lambda),
$$

where, e.g.,

$$
H(s, \lambda)=\int_{0}^{\infty} e^{-\lambda t} h(s, t) d t .
$$

Assuming that $a_{j}, j=1,2$, generate the same data $\{H(s, \lambda), Z(s, \lambda)\}_{\forall s \in S_{2}, \forall \lambda>0}$, one derives for

$$
w=v_{1}-v_{2}
$$


the problem

$$
\left(\mathcal{L}_{1}-\lambda\right) w=\nabla \cdot\left(p \nabla v_{2}\right), \quad p:=a_{2}-a_{1},\left.\quad w\right|_{S}=0,\left.\quad a_{1} v_{1 N}\right|_{S_{2}}=\left.a_{2} v_{2 N}\right|_{S_{2}} .
$$

Multiply (17) by an arbitrary element of $\mathcal{N}_{1}$, where

$$
\mathcal{N}_{j}=\left\{\varphi:\left(\mathcal{L}_{j}-\lambda\right) \varphi=0 \text { in } D,\left.\quad \varphi\right|_{S_{1}}=0\right\}, \quad j=1,2,
$$

integrate by parts, and get

$$
-\int_{D} p \nabla v_{2} \nabla \varphi d x+\int_{S} p v_{2 N} \varphi d s=\int_{S}\left(a_{1} w_{N} \varphi-a_{1} \varphi_{N} w\right) d s
$$

Using boundary conditions (17), one gets

$$
\int_{D} p \nabla \varphi \nabla v_{2} d x=0 \quad \forall \varphi \in \mathcal{N}_{1} \quad \forall v_{2} \in \mathcal{N}_{2}
$$

To complete the proof of Theorem 3, we use the following new version of Property C.

Lemma 1. The set $\left\{\nabla \varphi \cdot \nabla v_{2}\right\}_{\forall \varphi \in \mathcal{N}_{1}, \forall v_{2} \in \mathcal{N}_{2}}$ is complete in $L^{2}(D)$ for all sufficiently large $\lambda>0$.

We prove this lemma below, but first let us explain the claim made in the Introduction:

Claim: The results remain valid if the data are given not for all $t \geq 0$ but for $t \in[0, T+\varepsilon]$, where $\varepsilon>0$ is arbitrarily small.

This claim follows from the analyticity with respect to time of the solution $u(x, t)$ to problems (1)-(2) and (13)-(14) in a neighborhood of the ray $(T, \infty)$ for an arbitrary small $\varepsilon>0$. This analyticity holds if $a(s, t)$ and $h(s, t)$ vanish in the region $t>T$.

Proof of Lemma 1. It was proved in [2, pp.78-80], that the set $\left\{\nabla \psi_{1} \cdot \nabla \psi_{2}\right\}_{\forall \psi_{j} \in \mathcal{M}_{j}}$ is complete in $L^{2}(D)$, where

$$
\begin{gathered}
\mathcal{M}_{j}=\left\{\psi:\left(\mathcal{L}_{j}-\lambda\right) \psi_{j}=0\right\}, \\
\mathcal{L}_{j} \psi-\lambda \psi=\nabla \cdot\left(a_{j} \nabla \psi\right)-\lambda \psi,
\end{gathered}
$$

where $\lambda=$ const $>0,0<c \leq a_{j}(x) \leq C, a_{j}(x) \in H^{3}(D), j=1,2$, and $H^{\ell}(D)$ is the usual Sobolev space.

Lemma 1 will be proved if we prove that any $\psi_{j} \in \mathcal{M}_{j}$ can be approximated by an element $v_{j} \in \mathcal{N}_{j}$ with an arbitrary small error in the norm, generated by the bilinear form

$$
\int_{D}\left(a_{j} \nabla \psi_{j} \nabla \varphi+\lambda \psi_{j} \varphi\right) d x, \quad \varphi \in H^{1}(D) .
$$

The above norm is equivalent to the norm of $H^{1}(D)$ due to the assumption $0<c \leq$ $a_{j}(x) \leq C, \quad j=1,2$. 
Assuming that such an approximation is not possible, we can find a $\psi_{j} \in \mathcal{M}_{j}$ such that

$$
0=\int_{D}\left[a_{j}(x) \nabla \psi_{j} \nabla G_{j}(x, y)+\lambda \psi_{j} G_{j}(x, y)\right] d x \quad \forall y \in D^{\prime},
$$

because $G_{j}(x, y) \in \mathcal{N}_{j}$ for any $y \in D^{\prime}$. Here $G_{j}$ solves the problem

$$
\mathcal{M}_{j} G_{j}=-\delta(x-y) \text { in } \mathbb{R}^{3} \backslash S_{1},\left.\quad G_{j}\right|_{S_{1}}=0, \quad \lim _{|x-y| \rightarrow \infty} G_{j}(x, y)=0 .
$$

Integrating by parts in (20) and using the relation $G_{j} \in \mathcal{N}_{j}$, one gets

$$
0=\int_{S} a_{j} \psi_{j} G_{j N}(s, y) d s:=h(y), \quad \forall y \in D^{\prime} .
$$

Denote

$$
a_{j} \psi_{j}=\varphi, \quad G_{j}=G, \quad \mathcal{L}_{j}=\mathcal{L}
$$

Since

$$
(\mathcal{L}-\lambda) G=-\delta(x-y) \text { in } \mathbb{R}^{3} \backslash S_{1} \text { and }\left.G\right|_{S_{1}}=0,
$$

one can derive the relation

$$
\lim _{y \rightarrow p \in S_{1}} G_{N}(s, y)=\frac{\delta(s-p)}{a(s)},
$$

where $\delta(s-p)$ is the delta-function on $S_{1}$. We prove this relation later, but assuming that (23) holds we conclude from (21) that

$$
a_{j} \psi_{j}:=\varphi=0 \quad \text { on } \quad S_{1} .
$$

Consequently

$$
h(y)=\int_{S_{2}} \varphi(s) G_{N}(s, y) d s=0 \quad \forall y \in D^{\prime} .
$$

It follows from (25) that

$$
(\mathcal{L}-\lambda) h=0 \quad \text { in } \quad \mathbb{R}^{3} \backslash S_{2}, \quad h=0 \text { in } D^{\prime} .
$$

This and the uniqueness of the solution of the Cauchy problem for elliptic equation (26), imply

$$
h=0 \text { in } \mathbb{R}^{3} \backslash S_{2} \text {. }
$$

From (27) and the jump relation for the double-layer potential (25), we conclude that

$$
\varphi=0 \text { on } S_{2}
$$

From (24) and (28) it follows that $\varphi=0$ on $S$. Therefore $\psi_{j}=0$ on $S$ because $a_{j}>0$. Thus, $\psi_{j} \in \mathcal{M}_{j}$ and $\psi_{j}=0$ on $S$. This implies $\psi_{j}=0$ in $D$ for all sufficiently large $\lambda>0$. Thus, Lemma 1 is proved if (23) is established. 
Let us prove (23). Denote by $g$ the (unique) solution to the problem

$$
(\mathcal{L}-\lambda) g=-\delta(x-y) \text { in } \mathbb{R}^{3}, \quad \lim _{|x-y| \rightarrow \infty} g(x, y)=0 .
$$

Using Green's formula, we obtain from (22) and (29) the relation

$$
G(x, y)=g(x, y)-\int_{S_{1}} g(x, s) a(s) G_{N}(s, y) d s, \quad x, y \in S_{1}^{\prime}:=\mathbb{R}^{3} \backslash S_{1} .
$$

Taking $y \rightarrow p \in S_{1}$ and using the boundary condition (22), we derive:

$$
g(x, p)=\lim _{y \rightarrow p} \int_{S_{1}} g(x, s) a(s) G_{N}(s, y) d s \quad \forall x \in S_{1}^{\prime} .
$$

Since the set $\{g(x, p)\}_{\forall x \in S_{1}^{\prime}}$ is dense in $L^{2}\left(S_{1}\right)$, formula (31) implies (23).

Let us finally check the claim that the set $\{g(x, p)\}_{\forall x \in S_{1}^{\prime}}$ is dense in $L^{2}\left(S_{1}\right)$. If it is not dense, then there is an $f \in L^{2}\left(S_{1}\right)$ such that

$$
0=\int_{S_{1}} f(p) g(x, p) d p:=W(x), \quad \forall x \in S_{1}^{\prime} .
$$

The integral in (32) is a simple-layer potential $W(x)$ the density $f(p)$ of which must vanish because of the jump formula for the normal derivatives of $W$ across $S_{1}$. Thus the claim is proved. The proof of Lemma 1 is complete. Therefore Theorem 3 is proved.

Remark. If the conclusion of Lemma 1 remains valid for the set $\left\{\nabla \varphi \cdot \nabla v_{2}\right\}_{\forall \varphi \in \mathcal{N}_{1}, v_{2} \in \mathcal{N}_{2}}$, where $v_{2}$ is a single element of $\mathcal{N}_{2}$, possibly chosen in a special way, then the conclusion of Theorem 1 will be established for the data which is a single pair of data $\{a(s, t), b(s, t)\}_{\forall t \geq 0, \forall s \in S_{2}}$, where $a(s, t) \not \equiv 0$ is some function.

\section{References}

[1] Ramm, A. G., An inverse problem for the heat equation, J. Math. Anal. Appl., 264, N2, (2001), 691-697.

[2] Ramm, A. G. , Multidimensional Inverse Scattering Problems, Longman/Wiley, New York, 1992.

[3] Ramm, A. G., On completeness of the set of products of harmonic functions, Proc. of Amer. Math. Soc., 99, (1986), 253-256.

[4] Ramm, A. G., Inverse Problems, Springer, New York, 2005. 